\title{
Chemical composition of the outer halo globular cluster Palomar 15
}

\author{
Andreas Koch ${ }^{1}$, Siyi Xu (许偲艺) ${ }^{2}$, and R. Michael Rich ${ }^{3}$ \\ 1 Zentrum für Astronomie der Universität Heidelberg, Astronomisches Rechen-Institut, Mönchhofstr. 12, \\ 69120 Heidelberg, Germany \\ e-mail: andreas.koch@uni-heidelberg.de \\ 2 Gemini Observatory, Northern Operations Center, 670 N. A'ohoku Place, Hilo, HI 96720, USA \\ 3 University of California Los Angeles, Department of Physics \& Astronomy, Los Angeles, CA, USA
}

Received 14 March 2019 / Accepted 24 May 2019

\begin{abstract}
Globular clusters (GCs) in the outer Milky Way halo are important tracers of the assembly history of our Galaxy. Only a few of these objects show spreads in heavier elements beyond the canonical light-element variations that have essentially been found throughout the entire Galactic GC system, suggesting a more complex origin and evolution of these objects. Here, we present the first abundance analysis of three red giants in the remote $\left(R_{\mathrm{GC}}=38 \mathrm{kpc}\right)$ outer halo GC Palomar 15, based on medium-resolution spectra obtained with the Keck/ESI instrument. Our results ascertain a low iron abundance of $-1.94 \pm 0.06$ dex with no evidence of any significant abundance spreads, although this is based on low number statistics. Overall, abundance ratios of 16 species were measured, including carbon, $\mathrm{Na}, \mathrm{Al}, \alpha$-peak $(\mathrm{Mg}, \mathrm{Si}, \mathrm{Ca}, \mathrm{Ti})$ and $\mathrm{Fe}$-peak ( $\mathrm{Sc}, \mathrm{V}, \mathrm{Cr}, \mathrm{Fe}, \mathrm{Co}, \mathrm{Ni})$ elements, and the three neutron-capture elements $\mathrm{Sr}, \mathrm{Ba}$, and $\mathrm{Eu}$. The majority of abundances are compatible with those of halo field stars and those found in other GCs in the outer and inner halos at similar metallicity. Pal 15 is enhanced to $[\mathrm{Mg} / \mathrm{Fe}]=0.45 \mathrm{dex}$, while other $\alpha$-elements, $\mathrm{Ca}$ and $\mathrm{Ti}$, are lower by $0.3 \mathrm{dex}$. Taking $\mathrm{Mg}$ as a representative for $[\alpha / \mathrm{Fe}]$, and coupled with the lack of any significant spread in any of the studied elements we conclude that Pal 15 is typical of the outer halo, as is bolstered by its chemical similarity to the benchmark outer halo cluster NGC 7492. One star shows evidence of elevated $\mathrm{Na}$ and $\mathrm{Al}$ abundances, hinting at the presence of multiple stellar populations in this cluster.
\end{abstract}

Key words. Galaxy: abundances - Galaxy: evolution - globular clusters: individual: Palomar 15 - globular clusters: general Galaxy: halo

\section{Introduction}

Globular clusters (GCs), in particular those at large Galactocentric radii, are palmary objects for tracing the formation and structure of the Milky Way (MW). An inner-outer dichotomy as seen in various stellar tracers in the MW halo (Searle \& Zinn 1978; Hartwick 1987; Carollo et al. 2007) is also present in the nearby galaxy M 31 (Koch et al. 2008) and predicted by models of structure formation (Cooper et al. 2013; Pillepich et al. 2015). Here the outer halo GCs are vital study cases since some of these remote systems tend to be a few Gyr younger than inner halo clusters of the same metallicity, as is reflected, for instance, in the morphologies of their horizontal branches (Stetson et al. 1999; Catelan et al. 2001; Marín-Franch et al. 2009). This diversity in ages has often been interpreted as a sign of their accretion origin. Other distant GCs are, however, coeval with their old, metal-poor inner halo counterparts (Dotter et al. 2011), and many of the outer halo clusters are also chemically indistinguishable from the inner ones (Koch et al. 2009; Koch \& Côté 2010).

The relevance of the effects within GCs has gained major attention through their complex stellar populations (e.g., Gratton et al. 2012, and references therein) and the presence of pronounced light-element variations (e.g., Kayser et al. 2008; Carretta et al. 2009), all of which have caused a change in the canonical picture of "simple" GC formation (as comprehensively reviewed by Bastian \& Lardo 2018). Spreads in iron and some heavy chemical elements, however, have so far only been reported for a minority of mainly massive stellar systems, which suggests a different origin, possibly as parts of former dwarf galaxies (e.g., Mucciarelli et al. 2012; Kacharov et al. 2013; Marino et al. 2015; Johnson et al. 2017; Piatti \& Koch 2018).

Here we report on the first chemical abundance study of red giant branch stars in the outer halo GC Palomar 15 (herafter Pal 15). Pal 15 is an old GC $(13 \pm 0.5 \mathrm{Gyr}$; Dotter et al. 2011) situated at a Galactocentric radius of $38.4 \mathrm{kpc}$, making it one of the most remote GC satellites to the MW. Only ten GCs in the catalog of Galactic GCs of Harris (1996, 2010 version) are located at even further distances. While previous studies at low spectral resolution asserted a low metallicity of -2 dex (Da Costa \& Armandroff 1995), no detailed chemical abundances have been determined in this object.

This paper is organized as follows. In Sect. 2 we introduce the data acquisition and reduction. We describe the ensuing abundance analysis in Sect. 3, before turning to the results in Sect. 4. In Sect. 5 we compare our findings for Pal 15 with other outer halos GCs beyond $20 \mathrm{kpc}$, and we summarize our findings in Sect. 6.

\section{Observations and data reduction}

As Pal 15 is a remote, faint system we selected the brightest red giant candidates from the Hubble Space Telescope (HST) photometry of Dotter et al. (2011), complemented by infrared magnitudes from the Two Micron All Sky Survey (2MASS; Cutri et al. 2003). The respective color-magnitude diagram (CMD) is shown in Fig. 1, and in Table 1 we summarize the targets' main properties. Here we note that all our targets 

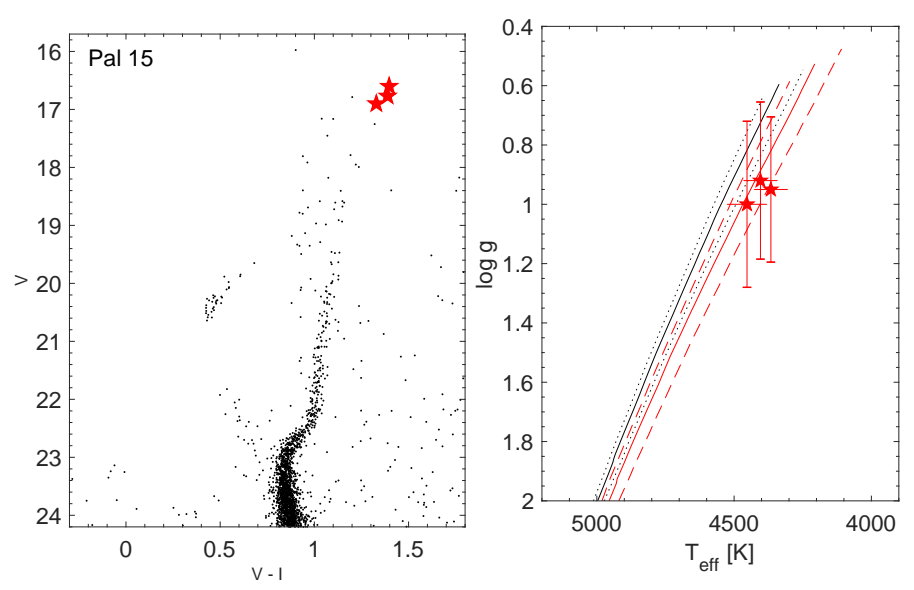

Fig. 1. Left panel: CMD of the target stars using the HST photometry of Dotter et al. (2011). Right panel: Kiel diagram with the spectroscopic parameters and old $(12.4 \mathrm{Gyr})$, metal-poor $(-2.2,-2.0,-1.8)$, Dartmouth isochrones (Dotter et al. 2008) with $\alpha$-enhancement (red lines) and without (black lines). Our targets are indicated as red stars.

are too faint to be extracted from Gaia DR2 (Gaia Collaboration 2018) so that no useful parallaxes could be extracted.

Our observations were carried out during the night of May 17, 2013, using the Echellette Spectrograph and Imager (ESI) instrument (Sheinis et al. 2002) at the Keck II telescope at Mauna Kea, Hawai'i. We employed a slit width of $0.5^{\prime \prime}$, yielding an intermediate resolving power of $R=8000$. Our data were reduced in a similar manner to Hansen et al. (2016a), by using the ESI-specific package within the $\mathrm{MAKEE}^{1}$ data reduction tool. As a result, the final spectra cover a full wavelength range of 4550-9000 $\AA$ and their signal-to-noise ratios (S/N) are in the range of 55-75 per pixel across the full spectrum.

All targets have already been classified as members in the kinematic study of Peterson \& Latham (1989), which we confirmed here by our radial velocity measurements using IRAF's fxcor tool against a synthetic red giant star template. For the three Pal 15 stars we find a mean heliocentric velocity and velocity dispersion of $72.4 \pm 1.0 \mathrm{~km} \mathrm{~s}^{-1}$ and $1.6 \pm 0.8 \mathrm{~km} \mathrm{~s}^{-1}$. These values are consistent with the findings of Peterson \& Latham (1989), and the latter, lower value is fully in line with the GC's low mass at $M_{V}=-5.5$ (Peterson \& Latham 1989; Pryor \& Meylan 1993).

\section{Abundance analysis}

Given the low resolution of our spectra, detailed equivalent width measurements and the ensuing excitation and ionization balances are inadequate for an accurate stellar parameter determination. Thus, we employed a two-step process for the abundance measurements.

\subsection{SP_Ace ( $T_{\text {eff }}, \log g,[\mathrm{Fe} / \mathrm{H}],[\mathrm{Mg}, \mathrm{Si}, \mathrm{Ca}, \mathrm{Sc}, \mathrm{Ti}, \mathrm{V}, \mathrm{Cr}, \mathrm{Co}$, $\mathrm{Ni} / \mathrm{Fe}])$}

First we used the SP_Ace code (Boeche \& Grebel 2016) to obtain the stars' effective temperatures and surface gravities using empirical curves of growth for a vast number of atomic

\footnotetext{
1 MAKEE was developed by T. A. Barlow specifically for reduction of Keck HIRES and ESI data. It is freely available on the World Wide Web at the Keck Observatory home page, https://www2 . keck. hawaii. edu/inst/common/makeewww/
}

lines across the entire spectral range. From these parameters the microturbulence was extracted by using the empirical formula provided in Boeche \& Grebel (2016). Furthermore, SP_Ace returns $\mathrm{Fe}$ abundances and chemical element ratios of several $\alpha$-elements (Mg,Si,Ca,Ti) and Fe-peak species ( $\mathrm{Sc}, \mathrm{V}, \mathrm{Cr}, \mathrm{Co}, \mathrm{Ni}$ ). The typical temperature errors as returned by SP_Ace are $80 \mathrm{~K}$ and the surface gravity could be determined to within 0.25 dex, leading to a typical uncertainty on the microturbulence of $0.25 \mathrm{~km} \mathrm{~s}^{-1}$. Color-temperature calibrations such as the $(V-K)$ relations of Ramírez \& Meléndez (2005) return $T_{\text {eff }}$ values that are $\sim 130 \mathrm{~K}$ warmer than our values, with a $1 \sigma$ deviation of $120 \mathrm{~K}$. This fair agreement reinforces our use of SP_Ace for deriving the stellar parameters of our sample.

All best-fit parameters are listed in Table 2, while Table 3 contains the abundance ratios determined by SP_Ace. Here we list the abundances as $[\mathrm{X} / \mathrm{Fe}]$ together with the $1 \sigma$ line-to-line scatter returned by SP_Ace, and the numbers of lines used in the analysis. Several examples of spectral regions around absorption lines of $\alpha$-elements used by SP_Ace are shown in Fig. 2 with the best synthesis in comparison with the observed spectrum of star 11726.

\subsection{Spectral synthesis with MOOG ([C,Sr,Ba,Eu/Fe])}

The stellar parameters derived above were then used to create model atmospheres by interpolating Kurucz's 1D 72-layer, plane-parallel, line-blanketed model grid without convective overshoot $^{2}$, where we assumed that local thermodynamic equilibrium holds for all species. This model grid further incorporated the $\alpha$-enhanced opacity distributions, AODFNEW (Castelli \& Kurucz 2003), as prompted by the $\alpha$-enhancement of the stars measured by SP_Ace.

For all further analyses, we used the 2014 version of the stellar abundance code MOOG (Sneden 1973) to determine abundances of a number of species via spectral synthesis. The line list for this purpose is based on Koch et al. (2016) with further additions from Hansen et al. (2012, 2013). All abundances thus determined were placed on the solar, photospheric scale of Asplund et al. (2009). This procedure was proved successful on medium-resolution ESI-spectra by Lai et al. (2009).

\section{Results}

Table 3 lists the abundance ratios we measured either from SP_Ace or from our spectral syntheses, where the last column details the method used for abundance determination.

\subsection{Iron}

Previous studies have established the metal-poor nature of Pal 15 by CMD fitting (Dotter et al. 2011) and from low-resolution CaT spectroscopy (Da Costa \& Armandroff 1995), both of which found an $[\mathrm{Fe} / \mathrm{H}]$ of -2 dex. This is confirmed by our spectroscopic study. From our three stars, we find a mean Fe-abundance of $-1.94 \pm 0.06$ with a $1 \sigma$ dispersion of $0.09 \pm 0.11$ dex determined in a maximum-likelihood approach. This low value is in line with the overall low metallicities of GCs in the outer halo, which peak at a mean $[\mathrm{Fe} / \mathrm{H}]$ of -1.7 dex beyond $20 \mathrm{kpc}$. In turn, about three objects at these large distances lie toward the lower cutoff of the GC metallicity distribution below -2 dex. Thus, Pal 15 is a typical representative of the outer halo population.

\footnotetext{
2 http://kurucz.harvard.edu/grids.html
} 
Table 1. Details of targets and observations.

\begin{tabular}{rccccccccc}
\hline \hline & $\begin{array}{c}\text { Alternate } \\
\text { ID }^{(a)}\end{array}$ & $\begin{array}{c}\alpha \\
\text { ID }^{(b)}\end{array}$ & $\begin{array}{c}\delta \\
(\mathrm{J} 2000.0)\end{array}$ & $\begin{array}{c}V \\
{[\mathrm{~J} 2000.0]}\end{array}$ & $\begin{array}{c}I \\
{[\mathrm{mag}]}\end{array}$ & $\begin{array}{c}K \\
{[\mathrm{mag}]}\end{array}$ & $\begin{array}{c}t_{\mathrm{exp}} \\
{[\mathrm{mag}]}\end{array}$ & $\begin{array}{c}S / N \\
{[\mathrm{~s}]}\end{array}$ & $\begin{array}{c}v_{\mathrm{HC}} \\
{\left[\mathrm{pixel}^{-1}\right]}\end{array}$ \\
\hline 3584 & SC-3 & $16: 59: 52.15$ & $-00: 33: 23.14$ & 16.601 & 15.204 & 12.969 & $3 \times 900$ & 55 & $72.1 \pm 0.6$ \\
8469 & SC-4 & $16: 59: 51.90$ & $-00: 32: 53.81$ & 16.770 & 15.381 & 13.281 & $3 \times 1200$ & 73 & $74.7 \pm 0.7$ \\
11726 & SC-7 & $16: 59: 56.05$ & $-00: 32: 23.23$ & 16.902 & 15.573 & 13.462 & $3 \times 1300$ & 73 & $70.3 \pm 0.8$ \\
\hline
\end{tabular}

Notes. ${ }^{(a)}$ Identifications from Dotter et al. (2011). ${ }^{(b)}$ Cross-identifications from Peterson \& Latham (1989).

Table 2. Stellar parameters of the individual target stars based on SP_Ace.

\begin{tabular}{crrr}
\hline \hline Parameter & \multicolumn{1}{c}{11726} & \multicolumn{1}{c}{8469} & \multicolumn{1}{c}{3584} \\
\hline$T_{\text {eff }}[\mathrm{K}]$ & $4453 \pm 140$ & $4404 \pm 118$ & $4366 \pm 115$ \\
$\log g$ & $1.00_{-0.37}^{+0.19}$ & $0.92_{-0.39}^{+0.14}$ & $0.95_{-0.31}^{+0.18}$ \\
$\xi\left[\mathrm{km} \mathrm{s}^{-1}\right]$ & $1.95 \pm 0.13$ & $1.92 \pm 0.11$ & $1.88 \pm 0.11$ \\
\hline
\end{tabular}

To date, Fe spreads have chiefly been found in rather massive GCs, which may point to more massive progenitors such as dwarf galaxies, while on the order of $5 \%$ of old galactic GC systems show significant intrinsic Fe spreads in excess of 0.05 dex (Johnson et al. 2015; Marino et al. 2015; Piatti \& Koch 2018). Considering its fairly low mass, at $M_{V}=-5.5 \mathrm{mag}$, Pal 15's lack of a significant Fe spread argues in favor of it being a regular GC with an ordinary enrichment history. In the following, we investigate this further by using additional tracers of chemical evolution.

\subsection{Carbon}

Carbon abundances were derived from spectral synthesis of the $\mathrm{CH} G$ band at $4300 \AA$; the typical fits (as determined in a $\chi^{2}$ sense) are shown in Fig. 3.

The results are shown in Fig. 4 in comparison with data for red giants in metal-poor $([\mathrm{Fe} / \mathrm{H}]<-2)$ GCs from Kirby et al. (2015). In all three stars of our study, the carbon abundance are very low, as is expected for such luminous red giants due to deep mixing that occurs during stellar evolution (Spite et al. 2005; Placco et al. 2014; Hansen et al. 2016b). Correcting for evolutionary effects would lead to increased values for the stars' intrinsic carbon abundances, but given the regularity of our results we do not pursue the investigation of carbon further.

\subsection{Light elements ( $\mathrm{Na}, \mathrm{Al})$}

Sodium abundances were measured from the strong $\mathrm{Na} \mathrm{D}$ resonance lines that we carefully deblended from interstellar contamination. These lines are very strong in our stars, with equivalent widths in excess of $300 \mathrm{~m} \AA$, so we advise caution with regard to the following interpretations. The resulting $\mathrm{Na}$ abundances were corrected for departures from local thermodynamic equilibrium (LTE; Lind et al. 2011a) and Table 3 lists the final non-LTE (NLTE) abundances.

One of the key signatures of GCs is a pronounced anticorrelation between $\mathrm{Na}$ and $\mathrm{O}$, indicating the presence of several stellar generations in each cluster. This also leads to the presence of light-element spreads that contrast the lack of any such variations in the heavy elements, safe for a few exceptions at the higher mass tail of GCs. Our spectral quality did not permit us to derive any oxygen abundances; for stars of our given stellar parameters, the commonly accessible O lines at 6300 or $7770 \AA$ are too weak to be detected.

Two of the stars in our sample have moderate enhancements in $\mathrm{Na}$ that are fully consistent with the properties of the first halolike generation that formed in the GC (Carretta et al. 2009). In turn, the brightest star shows a considerably higher abundance of $\sim 0.65 \mathrm{dex}$, which could indicate that it is a member of the second generation of Na-rich, O-poor stars, which also suggests that Pal 15 hosts multiple stellar generations as are seen in other GCs of similar age and mass (see, e.g., Fig. 9 in Bragaglia et al. 2017).

We synthesized the $\mathrm{Al}$ lines at $6696,6698 \AA$, and also obtained an estimate from the doublet at $8872 \AA$, but since they are very weak transitions, we urge caution when using the following results. We merely point out that star 11726 , which shows the highest $\mathrm{Na}$ abundance in our sample, also has an elevated $[\mathrm{Al} / \mathrm{Fe}]$ of $\sim 0.7$ dex. The other two stars (also poorer in $\mathrm{Na}$ ) have more moderate $\mathrm{Al}$ abundances, which provides further evidence that Pal 15 also shows light-element variations due to the presence of multiple populations (Carretta et al. 2018).

\section{4. $\alpha$-elements (Mg, Si, $\mathrm{Ca}, \mathrm{Ti})$}

The abundances of the key $\alpha$-elements are based on our analysis with SP_Ace and have been verified for the brightest star with MOOG. Our results are shown in Fig. 5, where we also overplot metal-poor halo field stars from Roederer et al. (2014) and the disk sample of Bensby et al. (2014). Furthermore, we show the mean abundances and abundance spreads of several outer halo GCs from the literature, which we discuss in more detail in Sect. 5.

By grouping together all four elements, we report a straight mean $[\alpha / \mathrm{Fe}]$ of $0.13 \pm 0.01 \mathrm{dex}$; this is a mild enhancement that is almost compatible with solar abundances. It is a rather low value for low-metallicity GCs, which typically follow the enrichment of the underlying Galactic component (e.g., Pritzl et al. 2005; Hendricks et al. 2016). Systematically lower values of $[\alpha / \mathrm{Fe}]$ with respect to halo field stars are a trademark signature of the low-mass dwarf galaxies (e.g., Matteucci \& Brocato 1990; Shetrone et al. 2001; Koch 2009; Tolstoy et al. 2009). This has spawned the notion that GCs that show depleted $\alpha$-abundances could be accreted objects that formerly belonged to more massive, complex systems such as these dwarfs (e.g., Brown et al. 1997; Cohen 2004; Villanova et al. 2013).

However, this simplistic combination of individual $\alpha$-elements is intrinsically problematic due to the different (explosive versus hydrostatic) burning phases producing these elements, leading to distinctive behaviors (e.g., Woosley \& Weaver 1995; Koch \& McWilliam 2011). We note the significantly elevated $[\mathrm{Mg} / \mathrm{Fe}]$ ratio of $0.46 \pm 0.05$ that lies on the plateau of halo field stars. This contrasts a strongly depleted $[\mathrm{Si} / \mathrm{Fe}]$ of $-0.10 \pm 0.03$. The reason for this lies in the weakness of the $\mathrm{Si}$ lines (typically below $16 \mathrm{m \AA}$ ) employed by $\mathrm{SP} \_$Ace coupled 
Table 3. Abundance ratios of the individual target stars and the GC mean.

\begin{tabular}{|c|c|c|c|c|c|c|c|c|c|c|c|c|}
\hline \multirow[t]{2}{*}{ Abundance ratio ${ }^{(a)}$} & \multicolumn{3}{|c|}{11726} & \multicolumn{3}{|c|}{8469} & \multicolumn{3}{|c|}{3584} & \multicolumn{2}{|c|}{ Pal 15} & \multirow[b]{2}{*}{ Note } \\
\hline & {$[\mathrm{X} / \mathrm{Fe}]$} & $\sigma$ & $\mathrm{N}$ & {$[\mathrm{X} / \mathrm{Fe}]$} & $\sigma$ & $\mathrm{N}$ & {$[\mathrm{X} / \mathrm{Fe}]$} & $\sigma$ & $\mathrm{N}$ & $\langle[\mathrm{X} / \mathrm{Fe}]\rangle$ & $\sigma$ & \\
\hline$[\mathrm{Fe} / \mathrm{H}]$ & -1.94 & 0.05 & 355 & -1.99 & 0.05 & 352 & -1.90 & 0.04 & 344 & $-1.94 \pm 0.02$ & $0.04 \pm 0.02$ & SP_Ace \\
\hline$[\mathrm{C} / \mathrm{Fe}]$ & -1.11 & +0.22 & 1 & -1.41 & +0.12 & 1 & -0.89 & +0.27 & 1 & $-1.19 \pm 0.25$ & $<0.01$ & Synth \\
\hline$[\mathrm{Na} / \mathrm{Fe}]^{(b)}$ & 0.66 & 0.01 & 2 & 0.26 & 0.13 & 2 & 0.10 & $\begin{array}{l}-0.40 \\
0.16\end{array}$ & 2 & $0.36 \pm 0.14$ & $0.23 \pm 0.10$ & Synth \\
\hline$[\mathrm{Mg} / \mathrm{Fe}]$ & 0.41 & 0.21 & 5 & 0.47 & 0.18 & 6 & 0.48 & 0.19 & 4 & $0.45 \pm 0.18$ & $<0.01$ & SP_Ace \\
\hline$[\mathrm{Al} / \mathrm{Fe}]$ & 0.70 & 0.30 & 4 & 0.30 & 0.30 & 4 & $<0.50$ & $\ldots$ & 4 & $0.50 \pm 0.17$ & $<0.01$ & Synth \\
\hline$[\mathrm{Si} / \mathrm{Fe}]$ & $-0.04:$ & 0.18 & 17 & $-0.16:$ & 0.23 & 15 & -0.15 & 0.20 & 15 & $-0.11 \pm 0.03$ : & $0.03 \pm 0.04$ & SP_Ace \\
\hline$[\mathrm{Ca} / \mathrm{Fe}]$ & 0.13 & 0.07 & 26 & 0.12 & 0.06 & 26 & 0.17 & 0.05 & 26 & $0.14 \pm 0.01$ & $0.02 \pm 0.01$ & SP_Ace \\
\hline$[\mathrm{Sc} / \mathrm{Fe}]$ & 0.00 & 0.11 & 15 & 0.04 & 0.13 & 15 & 0.09 & 0.09 & 14 & $0.05 \pm 0.02$ & $0.03 \pm 0.02$ & SP_Ace \\
\hline$[\mathrm{Ti} / \mathrm{Fe}]$ & -0.01 & 0.10 & 58 & 0.01 & 0.10 & 54 & 0.11 & 0.07 & 56 & $0.04 \pm 0.03$ & $0.05 \pm 0.02$ & SP_Ace \\
\hline$[\mathrm{V} / \mathrm{Fe}]$ & -0.23 & 0.15 & 22 & -0.26 & 0.23 & 24 & -0.25 & 0.17 & 28 & $-0.23 \pm 0.03$ & $<0.01$ & SP_Ace \\
\hline$[\mathrm{Cr} / \mathrm{Fe}]$ & -0.08 & 0.19 & 24 & -0.07 & 0.18 & 24 & -0.11 & 0.15 & 23 & $-0.09 \pm 0.17$ & $<0.01$ & SP_Ace \\
\hline$[\mathrm{Co} / \mathrm{Fe}]$ & -0.14 & 0.20 & 20 & 0.01 & 0.28 & 24 & -0.04 & 0.18 & 23 & $-0.04 \pm 0.04$ & $<0.01$ & SP_Ace \\
\hline$[\mathrm{Ni} / \mathrm{Fe}]$ & -0.11 & 0.09 & 62 & -0.08 & 0.08 & 60 & -0.04 & 0.06 & 58 & $-0.08 \pm 0.02$ & $0.03 \pm 0.01$ & SP_Ace \\
\hline$[\mathrm{Sr} / \mathrm{Fe}]$ & 0.60 & 0.15 & 1 & 0.55 & 0.20 & 1 & 0.40 & 0.25 & 1 & $0.55 \pm 0.20$ & $<0.01$ & Synth \\
\hline$[\mathrm{Ba} / \mathrm{Fe}]$ & 0.54 & 0.19 & 4 & 0.45 & 0.25 & 4 & 0.62 & 0.27 & 4 & $0.54 \pm 0.10$ & $<0.01$ & Synth \\
\hline$[\mathrm{Eu} / \mathrm{Fe}]$ & 0.40 & 0.20 & 2 & 0.40 & 0.40 & 2 & 0.50 & 0.40 & 2 & $0.42 \pm 0.13$ & $<0.01$ & Synth \\
\hline
\end{tabular}

Notes. ${ }^{(a)}$ All abundance ratios are stated as the mean value, $1 \sigma$ standard deviation, and number of lines used. $[\mathrm{C} / \mathrm{Fe}]$ was measured from the molecular $G$ band. Abundance errors from spectral synthesis are based on a weighted average after visual quality control of the individual lines. ${ }^{(b)}$ NLTE value.

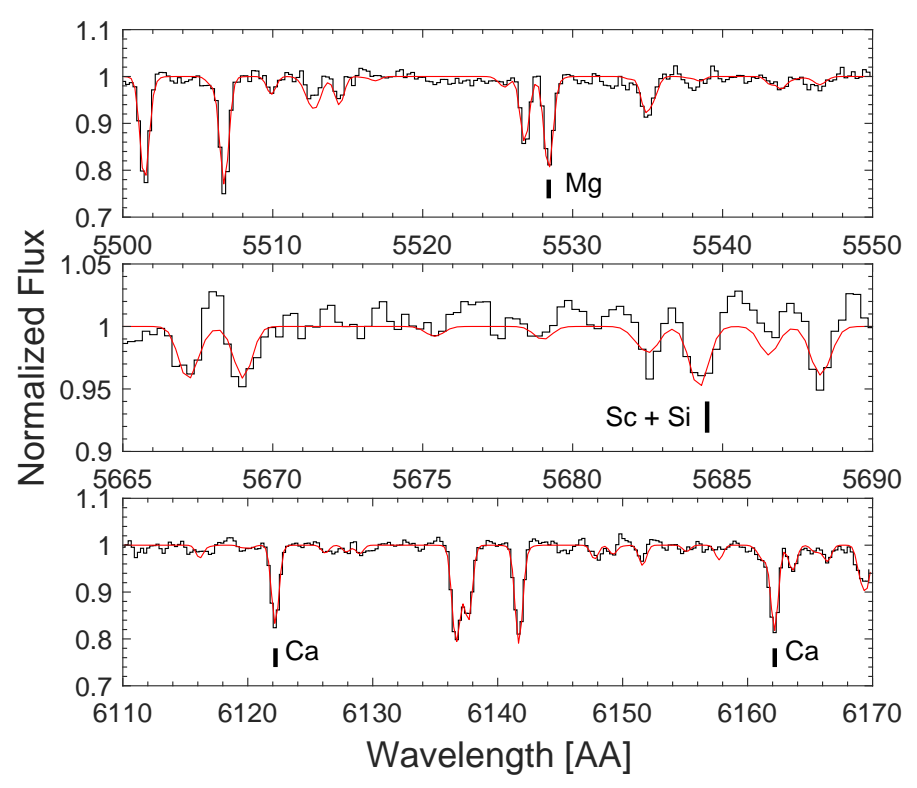

Fig. 2. Sample regions around $\alpha$-element lines that were used by SP_Ace. The best model computed by SP_Ace is shown in red superimposed on the observed spectrum of star 11726.t.

with the intermediate resolving power of our spectra. Here, we note that the code does not contain transitions in the wavelength range of $6860-8400 \AA$, whereas redder wavelengths up to $8800 \AA$ are again considered. Therefore, this overinterpretation of the low Si abundance should be taken with a grain of salt.

The explosive element abundances for $\mathrm{Ca}$ and $\mathrm{Ti}$ take values in between the enhanced (hydrostatic) $\mathrm{Mg}$ and depleted $\mathrm{Si}$, and they lie toward the lower boundaries outlined by the halo field stars. Finally, we note that Dotter et al. (2011) found that an $\alpha$-enhancement of 0.4 dex in their isochrones best represents the cluster's CMD (right panel of Fig. 1). Taking $\mathrm{Mg}$ as a prime $\alpha$-element that, as a significant electron donor, strongly

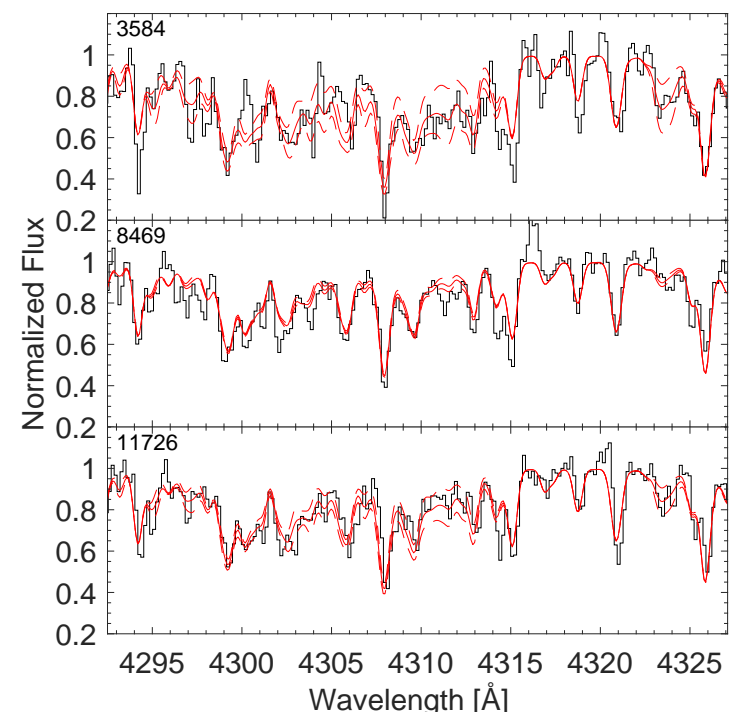

Fig. 3. Spectra of the three red giants near the $\mathrm{CH} G$ band. The red lines show the best-fit spectrum and the synthesis of the error margins as dashed lines.

affects stellar atmospheres and therefore the appearance of the isochrones, the concordance between our reported $[\mathrm{Mg} / \mathrm{Fe}]$ and the CMD fitting corroborates the enhancement of Pal 15 and its place on the Mg plateau that is seen for the majority of halo stars and GCs.

\subsection{Fe-peak elements (Sc, V, Cr, Co, Ni)}

Given the fortuitous spectral range of our data, we were able to extract abundance ratios for five $\mathrm{Fe}$-peak elements using SP_Ace. They are indicated in Fig. 6 in comparison with the same halo data as mentioned above, with added measurements in the Galactic disk from Battistini \& Bensby (2015). 


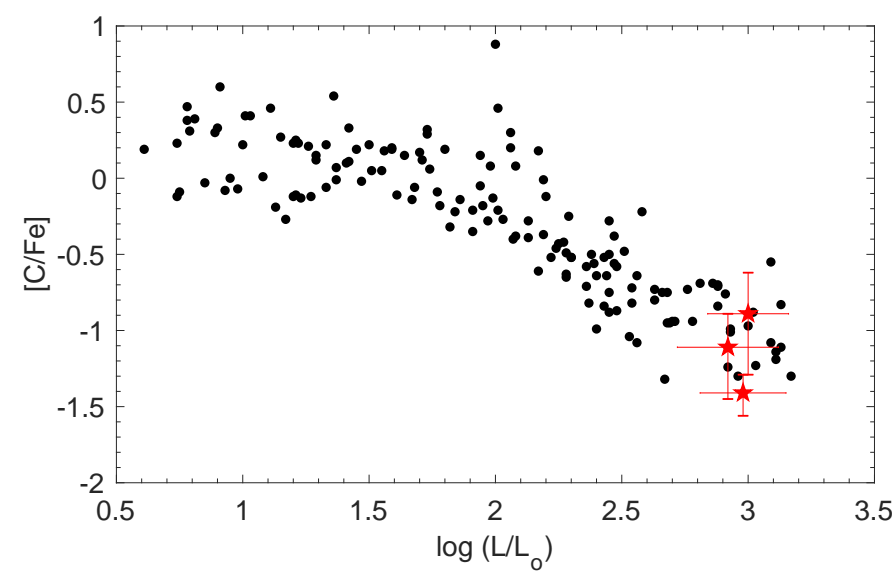

Fig. 4. Carbon abundances of red giants in Pal 15 (red stars) and metalpoor halo GCs (black dots; Kirby et al. 2015). The latter are not corrected for evolutionary effects in this representation.

The Fe-peak elements are synthesized in supernovae (SNe) of both Type Ia and II, while at higher, solar metallicity, there is a preponderance of SNe Ia in the production of these elements (e.g., Woosley \& Weaver 1995; Kobayashi et al. 2006). There was no surprise from the abundance ratios we derived in Pal 15 stars in comparison with halo stars of similarly low metallicity, and we note a remarkable overlap of our GC stars with the abundances of the stellar halo. We note, however, that none of our results was corrected for departures from LTE, which would mainly have some effects on $\mathrm{Cr}$ (e.g., Bergemann \& Cescutti 2010) and can explain the decrease in the $\mathrm{Cr} / \mathrm{Fe}$ ratio in the halo with decreasing metallicity. Cobalt has mixed contributions from $\mathrm{SNe}$ of type Ia and II, while hypernovae are also viable channels (Timmes et al. 1995; Kobayashi et al. 2006). As we did for $\mathrm{Cr}$, here we did not correct our $\mathrm{Cr}$ results for NLTE (Bergemann et al. 2010). The values for $[\mathrm{Co} / \mathrm{Fe}]$ in Pal 15 are entirely in line with those in halo field stars, and little variation in this element is seen in the other GCs.

\subsection{Neutron-capture elements (Sr, Ba, Eu)}

Abundances for three tracers of the neutron-capture processes were determined by using spectral synthesis. Here the mean abundance and errors were estimated via the best matching synthetic fit for each absorption feature used, weighted by the quality (in terms of $\mathrm{S} / \mathrm{N}$ and continuum setting) of the respective lines. The resulting abundance ratios are shown in Fig. 7, where we overplot the halo comparison samples from Hansen et al. (2012) and Roederer et al. (2014) and disk stars from Battistini \& Bensby (2016).

Strontium abundances were determined from the resonance line at $4215 \AA$, the usefulness of which even at low resolution has been demonstrated by, for instance, Stanford et al. (2010) and Koch et al. (2017). As above, we did not correct our results for $\mathrm{Sr}$ for departures from LTE as they are negligible at the stellar parameters of our stars (Hansen et al. 2013). As Fig. 7 indicates, the $[\mathrm{Sr} / \mathrm{Fe}]$ ratio in $\mathrm{Pal} 15$ grazes the higher values of the distribution of halo stars; however, the halo stars show a broad scatter at these low metallicities themselves. While the data in other GCs are sparse, the three outer halo GCs with measured $\mathrm{Sr}$ abundances are characterized by solar to subsolar values.

Our $\mathrm{Ba}$ measurements are based on synthesizing the typically well-described lines at 4554, 5853, 6141, and $6496 \AA$. Here we adopted the solar-scaled isotopic ratio, but we note

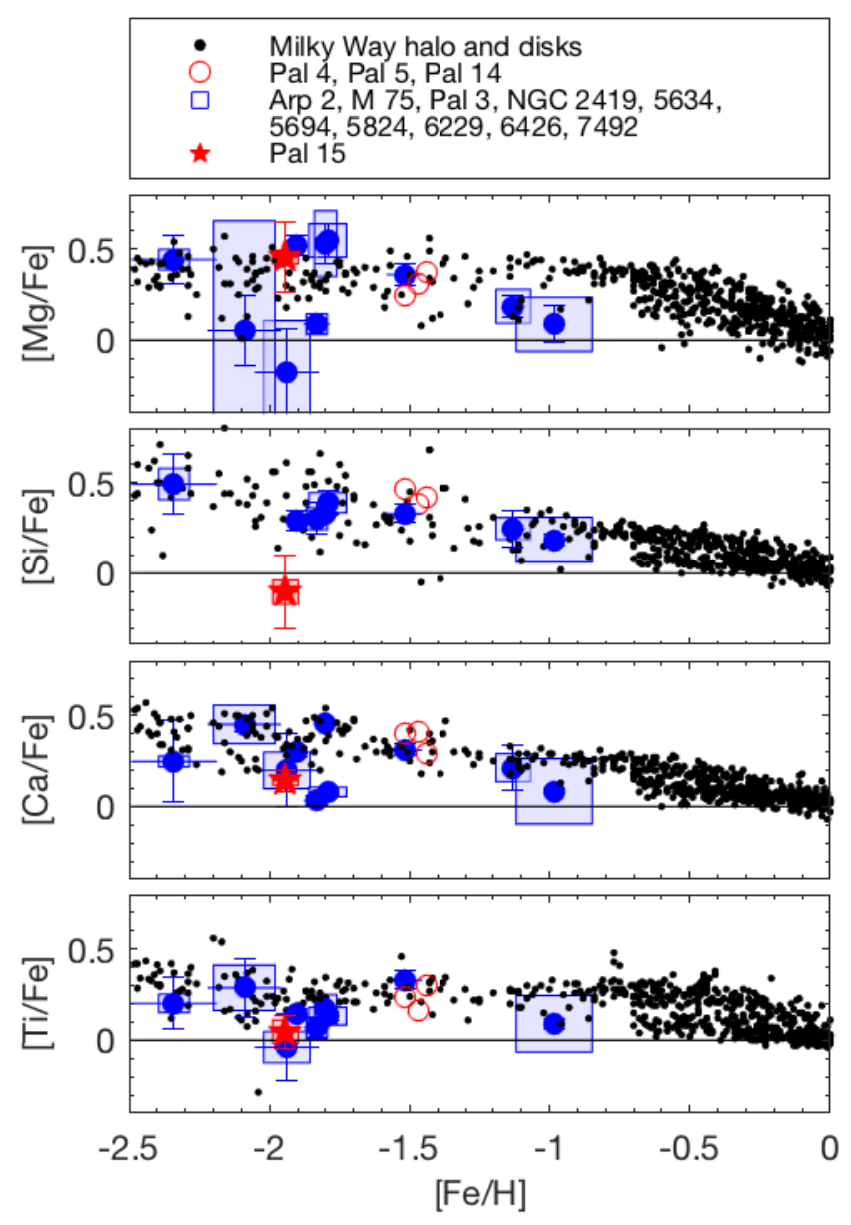

Fig. 5. Abundances of the $\alpha$-elements for Pal 15 (red star) and the reference outer halo GCs. Here, red open circles show GCs with mean abundances derived from co-added spectra. The blue boxplots refer to the observed $1 \sigma$ spread in a given GC (extent of the boxes in either dimension), while the inlaid error bars depict the mean abundance error on the respective measurements.

that switching to pure $r$ - or pure $s$-ratios (Sneden et al. 2008) essentially leaves our results of these moderately metal-poor stars unchanged. The resulting mean abundance of $\sim 0.57 \mathrm{dex}$ appears rather high in the Galactic context. On the other hand, (Andrievsky et al. 2009) estimated NLTE corrections on the order of 0.2 dex for stars with similar stellar parameters to those in our sample. These values would correct our inferred Ba abundances downward to reasonable levels when compared to halo and GC stars.

Finally, we estimated Eu-abundances from the $6645 \AA$ line, which is very weak, however, leading to typical uncertainties at the $0.2-0.3$ dex level. In turn, the blue $4129 \AA$ line yielded additional information and we report a visually weighted average of both features as our final Eu abundance in the following. At $\sim 0.4$ dex, the respective value is consistent with the majority of halo field stars and Galactic GCs with reported abundances.

\section{Comparison with outer halo GCs beyond $20 \mathrm{kpc}$}

In the following, we contrast our measurements in Pal 15 with the abundance ratios found in other outer halo GCs; we restrict the sample to the objects at Galactocentric distances beyond $\sim 15 \mathrm{kpc}$. With few exceptions, all of these are moderately metal poor at an $[\mathrm{Fe} / \mathrm{H}]$ of about $-1.5 \mathrm{dex}$ and most 

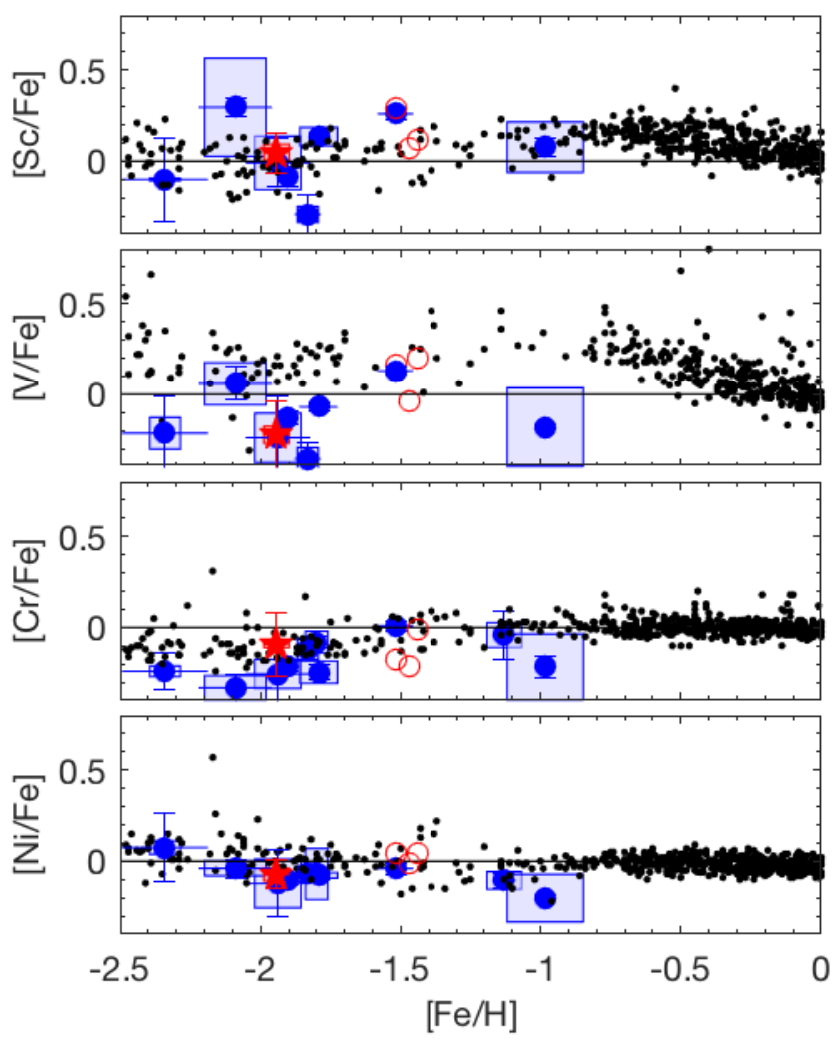

Fig. 6. Same as Fig. 5, but for the Fe-peak elements Sc, V, Cr, and Ni.

show $\alpha$-enhancements that are typical of the Galactic halo at these metallicities; we discuss deviations in Sect. 5.1. Combined with their, on average, younger ages (e.g., Stetson et al. 1999; Marín-Franch et al. 2009) this argues in favor of them being accreted objects, adding to the complexity of the formation of the outer halo.

Some of the reference GCs in the literature claim to show broad spreads in some abundances past the key $\mathrm{CN} / \mathrm{CH}$ or $\mathrm{Na}-\mathrm{O}$ variations. In particular, proton-capture reactions in the progenitors of the second stellar populations tend to produce a mild Mg-Al correlation, while spreads in some $s$-process elements are also seen, pointing to specific channels of enrichment from asymptotic giant branch (AGB) stars (Marino et al. 2015; Cordero et al. 2015). Furthermore, Fe spreads have been reported in about ten of the MW GCs studied in detail at high spectral resolutions. To this end, we show the abundance distribution of these clusters via boxplots, where the height and width of each box illustrates the observed $1 \sigma$ dispersion in a given abundance ratio and in the measured $\mathrm{Fe}$ abundance in the stellar samples (of typically a few to tens of stars). We note that the appearance as broad boxes does not imply that the GCs in question have significant intrinsic abundance spreads of that magnitude. Moreover, this illustrates the possible parameter space inhabited within the error bars. These error bars in the figures refer to the mean abundance error as stated in the literature. In this regard, error bars exceeding the box sizes indicate that the observed dispersions are mainly driven by observational errors, while those immured by the $\sigma$ box would suggest the presence of real intrinsic spreads. Individual cases are discussed below.

\subsection{Individual outer halo GCs}

For three of the objects, abundance ratios are only available from co-added spectra. That is, individual, low $\mathrm{S} / \mathrm{N}$ spectra

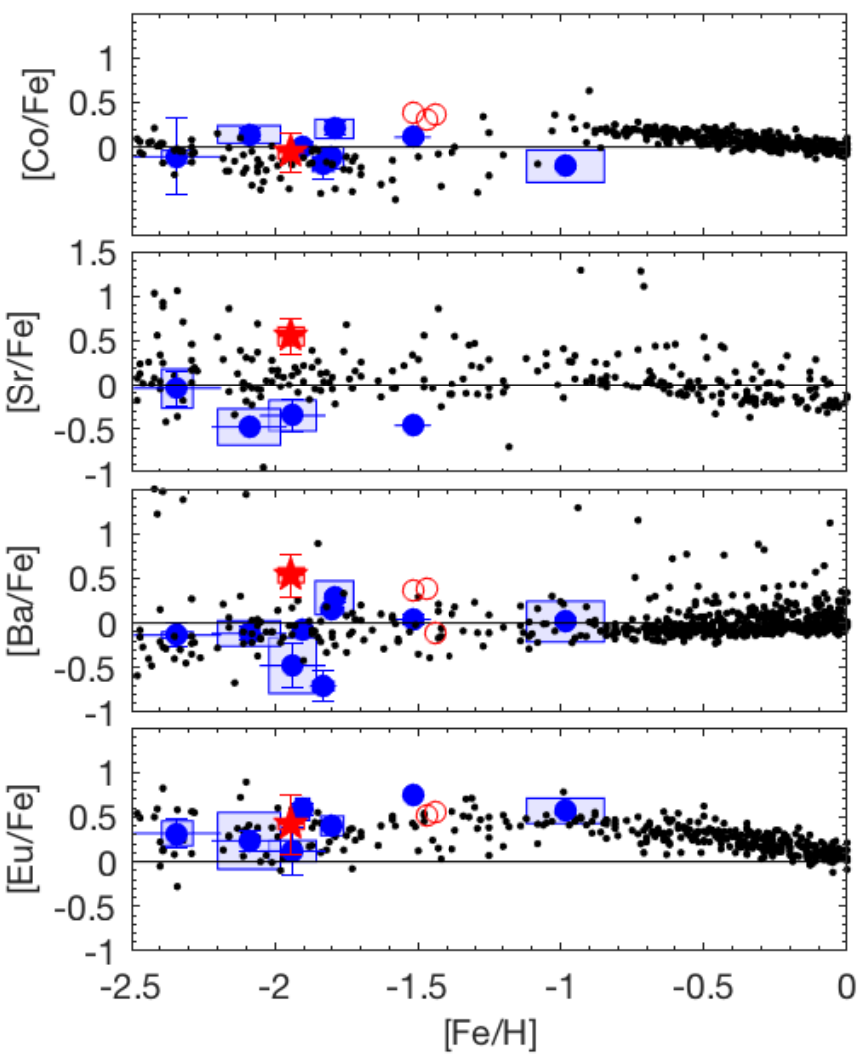

Fig. 7. Same as Fig. 5, but for Co and the neutron-capture elements Sr, $\mathrm{Ba}$, and $\mathrm{Eu}$.

of stars with known stellar parameters were combined into a master spectrum and were compared against a co-added synthetic spectrum with the same properties. This yields a mean abundance ratio of the GC in question. However, as pointed out by Koch \& Côté (2017), this method is highly insensitive to intrinsic abundance spreads in the GCs, and even the prominent light-element variations (e.g., in $\mathrm{Na}$ and $\mathrm{O}$ ) are undetectable to the $0.6 \mathrm{dex}$ level. These very remote systems comprise Pal $5\left(R_{\mathrm{GC}}=19 \mathrm{kpc}\right.$; Koch \& Côté 2017), Pal 4 $\left(R_{\mathrm{GC}}=111 \mathrm{kpc}\right.$; Koch \& Côté 2010), and Pal $14\left(R_{\mathrm{GC}}=77 \mathrm{kpc}\right.$; Çalışkan et al. 2012). Pal $3\left(R_{\mathrm{GC}}=96 \mathrm{kpc}\right)$ has both co-added and individual measurements available, which yield consistent results (Koch et al. 2009).

Among the objects with available high-resolution abundances from individual stars, the metal-rich $([\mathrm{Fe} / \mathrm{H}]=-1 \mathrm{dex})$ GC M75 $\left(R_{\mathrm{GC}}=15 \mathrm{kpc}\right)$ shows an indication of a spread in its Fe-abundance typical of its luminosity. Furthermore Kacharov et al. (2013) detected three generations of stars in abundance space, and variations in the masses of AGB polluters, leading to a broad spread in $s$-process elements between stars of either population.

NGC $6426\left(R_{\mathrm{GC}} \sim 15 \mathrm{kpc}\right)$ is one of the most metal-poor systems in the MW halo $([\mathrm{Fe} / \mathrm{H}]=-2.3 \mathrm{dex})$. While only based on four stars, Hanke et al. (2017) suggested abundance spreads, though marginal, in several elements, adding detections of a correlation between $\mathrm{Mg}, \mathrm{Si}$, and $\mathrm{Zn}$ to the pool of the canonical $(\mathrm{C}, \mathrm{N}, \mathrm{Na}, \mathrm{O}, \mathrm{Mg}, \mathrm{Al})$ light-element variations.

The metal-poor $([\mathrm{Fe} / \mathrm{H}] \sim-2 \mathrm{dex}) \operatorname{NGC} 5634\left(R_{\mathrm{GC}}=21 \mathrm{kpc}\right)$ is a GC that has been associated with the disrupted Sagittarius (Sgr) dwarf galaxy based on position and dynamical arguments (Law \& Majewski 2010), although its chemical abundances are indistinguishable from those of MW halo field stars 

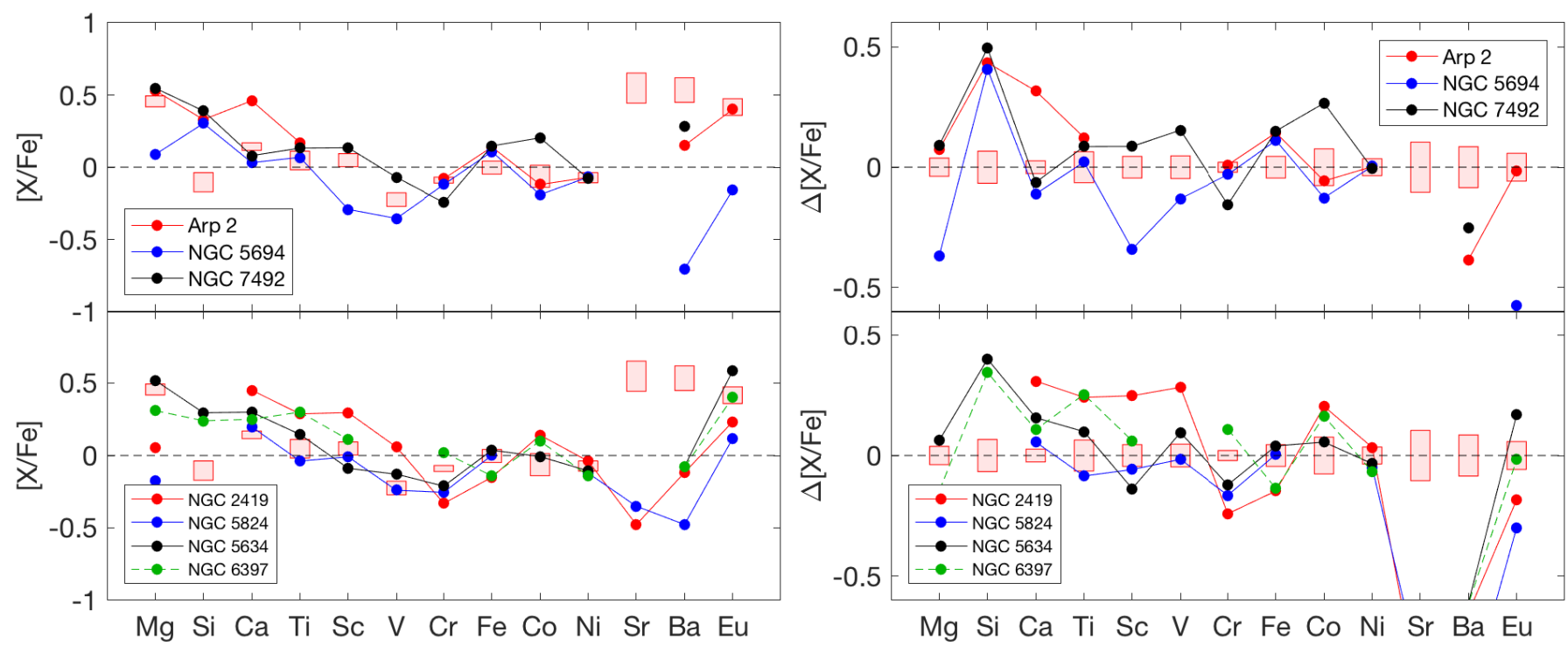

Fig. 8. Comparison of Pal 15 (red boxes, scaled to the respective abundance scatter) with seven GCs at similar metallicities. Left panels: abundance ratios. Right panels: difference $[\mathrm{X} / \mathrm{Fe}]_{\mathrm{GC}}-[\mathrm{X} / \mathrm{Fe}]_{\text {Pal15. }}$. To help readability, the figures are split into two panels each. Top panels: three objects at -1.8 dex, and bottom panels: remaining more metal-poor reference GCs. For iron, we show $[\mathrm{Fe} / \mathrm{H}]$ relative to the Pal 15 mean.

(Sbordone et al. 2015). Here we used the extended chemical abundance measurements from Carretta et al. (2017), who found no evidence of any significant abundance spread. Similarly, Arp 2 (at $21 \mathrm{kpc} ;[\mathrm{Fe} / \mathrm{H}]=-1.8)$ is a purported member of the Sgr system. Mottini et al. (2008) performed a high-resolution abundance study of this GC, although the small sample size of two stars prevents a detailed investigation of the question of abundance spreads.

NGC 5824 is an intriguing object at $R_{\mathrm{GC}}=26 \mathrm{kpc}$ whose nature is still disputed. For instance, Mucciarelli et al. (2018) measured a systematic difference in iron abundance between their AGB and RGB samples at the 0.1 dex level, which is, however, easily explained as measurement uncertainties. The same study ascertained the presence of an "extreme" $\mathrm{Mg}$-Al anticorrelation as typically seen in metal-poor and/or very massive clusters, and driven by the self-enrichment via massive AGB stars. The authors thus ruled out the possibility that NGC 5824 is the remnant of a disrupted dwarf galaxy. This is in stark contrast to previous morphological evidence (e.g., Kuzma et al. 2018) and the recent finding of Yuan et al. (2019) who, kinematically, identified this GC with the nuclear star cluster of the Cetus stream. In our figures, we include the comprehensive abundance measurements from Roederer et al. (2016), who reported on further abundance spreads in this cluster, but we note the overall more metal-rich mean found by those authors.

NGC $5694\left(R_{\mathrm{GC}}=29 \mathrm{kpc}\right)$ is of similar interest: for this GC, Mucciarelli et al. (2013) find remarkably low abundances of the $\alpha$-elements and several neutron-capture elements. While $\mathrm{Na}$ and $\mathrm{O}$ show the expected broad ranges due to the evolutionary anticorrelation, no other abundance spreads have been found. Based on the overall low mean abundances, these authors confirmed the hypothesis of Lee et al. (2006) that NGC 5694 is of extragalactic origin, which is also supported by the presence of an extratidal halo around this GC (Correnti et al. 2011).

Next, four stars in NGC $7492\left(R_{\mathrm{GC}}=25 \mathrm{kpc}\right)$ have been analysed by Cohen \& Melendez (2005), who find this outer halo GC to be chemically indistinguishable (including the neutron capture elements) from its inner halo counterparts such as M3 or M13. With the additions of the even more remote $\mathrm{Pal} 3$ and $\mathrm{Pal} 4$, which share the same chemical similarities (Koch et al. 2009; Koch \& Côté 2010), this bolsters the view of a homogeneous chemical history of the inner and outer halos at least through the formation epochs of these old systems, as witnessed by these metal-poor GCs. Furthermore, Cohen \& Melendez (2005) find no abundance spreads in this object safe from the expected variations in $\mathrm{Na}$ and $\mathrm{O}$.

NGC $6229\left(R_{\mathrm{GC}}=30 \mathrm{kpc}\right)$ is a rather metal-rich system $([\mathrm{Fe} / \mathrm{H}] \sim-1.1)$ with a modest dispersion of $0.06 \mathrm{dex}$ in iron (Johnson et al. 2017). Moreover, these spreads also permeate into the heavier elements. For instance, Johnson et al. (2017) find that 2 of 11 stars in their sample show enhanced La and $\mathrm{Nd}$ abundances that possibly correlate wth Fe. Furthermore, the stars in this GC show, overall, systematically lower $\mathrm{Na}$ and $\mathrm{Al}$ abundances, suggesting an accretion origin, possibly as the core of a disrupted dwarf galaxy. Given the similarity of NGC 6229 to two other GCs (M 75 and NGC 1851) with regard to many abundance ratios and other characteristics, Johnson et al. (2017) dubbed this system and its brethren an Fe-complex cluster, whose members are characterised by prolonged enrichment histories.

The most remote GC with reported individual highresolution abundances is NGC $2419\left(R_{\mathrm{GC}}=86 \mathrm{kpc}\right)$. It has been characterized as a bizarre object that not only exhibits a remarkable metallicity spread, but also significant spreads in certain light elements that have, to date, not been seen in other GCs (e.g., Cohen \& Kirby 2012). In particular, the distribution of $\mathrm{Mg}$ abundances is bimodal and strong variations in $\mathrm{K}$ and $\mathrm{Sc}$ have been found. Iron and calcium, on the other hand, are well behaved and show no significant spreads whatsoever. This cluster remains puzzling: while Cohen \& Kirby (2012) point out the resemblance of the $\mathrm{Mg}$ depletions with those seen in dwarf spheroidal galaxies and the lack of any nucleosynthetic sites that could simultaneously explain all of the abundance variations seen in this object, the lack of a significant Fe spreads contradicts this unique explanation. NGC 2419 remains unique in that it is like no other GC, and it may (or may not) be the core of an accreted dwarf galaxy. In the following figures, we use the abundance data from Mucciarelli et al. (2012) and Cohen \& Kirby (2012) for the heavier elements. 


\subsection{Comparison with $\mathrm{Pal} 15$}

Several GCs share individual abundance ratios with Pal 15, although none has the exact same pattern. In Fig. 8 we thus compare the abundance pattern of Pal 15 with those in the outer halo GCs discussed above. Here we note that, strictly speaking, our abundance ratios for certain elements should be corrected for the effects of NLTE. However, our targets are mainly red giants with small corrections. More importantly, here we are comparing our results with GCs of similar metallicity and targets of similar stellar parameters. Thus, all objects experience the same order of corrections, thereby minimizing systematic differences in the present comparative analysis.

Among the reference objects considered here, NGC 7492 shows a good resemblance, in a statistical sense, to the elements studied here. In particular, for 5 out of the 11 elements in common, the patterns of these two GCs agree to better than 0.1 dex. Among the elements that deviate more are those elements mainly derived from weak lines ( $\mathrm{Si}, \mathrm{V})$; $\mathrm{Cr}$, for which NLTE corrections may be relevant; and Ba. As Cohen \& Melendez (2005) characterized NGC 7492 as a typical halo cluster, the resemblance mentioned above suggests that Pal 15 is equally ordinary. While it appears tempting to associate NGC 7492 and Pal 15 as an Fe-complex cluster because they share the same chemical properties (Johnson et al. 2017), we note that neither of these objects shows any significant spread in the elements considered here. Their similarity attests to a certain degree of homogeneity in the outer halo population, at least as sampled by these GCs.

As a final comparison object we tested our results against the cluster NGC 6397, which has a similar metallicity (Koch \& McWilliam 2011), but is located in the inner halo at a mere $R_{\mathrm{GC}}=6 \mathrm{kpc}$. Here, we make a comparison with the NLTE abundances from Lind et al. (2011b). As the green dashed line in Fig. 8 shows, one-third of the elements in common between the two outer and inner halo GCs agree to better than 0.1 dex, emphasizing the complexity and variety of GCs that are not necessarily dependent on the inner or outer halo location (Milone et al. 2017).

\section{Discussion}

Our abundance analysis of three red giants in the outer halo GC Pal 15 has revealed no strong evidence of it being an accreted object, but rather suggests that it is an ordinary metal-poor halo cluster. Its similarity to another outer halo GC, NGC 7492, that has been shown to closely follow the outer halo population, indicates the chemical homogeneity of this Galactic component with respect to its non-accreted population. Taking $\mathrm{Mg}$ as a representative $\alpha$-element, we do not see a depletion that would be a key signature of an extragalactic accretion origin. Lower values are mainly seen in $\mathrm{Ca}$ and $\mathrm{Ti}$, which are, however, still compatible with the lower boundary of halo field and other GC stars. In order to resolve the seeming ambiguity between the different $\alpha$-elements, spectra at higher resolution of a larger sample of stars are needed, which is challenging given the GC's faintness.

The Fe-peak elements are slightly subsolar to solar and wellbehaved, which also holds for the few neutron-capture species we sampled. This is bolstered by the absence of any significant spread in any of the abundances studied. Pal 15 is a fairly faint system $\left(M_{V}=-5.5\right)$. Other objects of similar low luminosities and comparably old ages are known to have produced a Na-O anti-correlation (Bragaglia et al. 2017; Bastian \& Lardo 2018). While we see tentative evidence of a second generation of
Na-enhanced stars in Pal 15, this needs to be consolidated from higher resolution studies of a larger sample of stars.

The remainder on the list of known outer halo GCs beyond $20 \mathrm{kpc}$ (Harris 1996) without any published abundances from high-resolution spectroscopy, to the best of our knowledge, is surprisingly long: NGC 4147 (21 kpc), Pal 13 (27 kpc), AM 4 (28 kpc), Whiting 1 (35 kpc), Pal 2 (35 kpc), NGC 7006 (39 kpc), Pyxis (41 kpc), Koposov 1 and 2 (49 and $42 \mathrm{kpc}$ ), Eridanus $(95 \mathrm{kpc})$, and AM 1 (125 kpc). In some cases, low-resolution metallicity estimates are available from the near-infrared calcium triplet (e.g., Suntzeff et al. 1985), and for others the presence of multiple populations and light-element variations has been ascertained from photometric and low-resolution data (e.g., Harbeck et al. 2003; Gerashchenko \& Ananjevskaja 2018). Obtaining the full chemical abundance information for these systems is imperative for disentangling the formation history of outer halo and the occurrence of abundance variations as a function of GC mass and environment. For the most remote objects, this is hampered by the sparseness of the GCs and faintness of the accessible red giants, with the brightest member stars being fainter than $V=18 \mathrm{mag}$ (Hilker 2006), which renders detailed abundance measurements time-intensive.

Acknowledgements. A.K. acknowledges financial support from the Sonderforschungsbereich SFB 881 "The Milky Way System" (subprojects A03, A05, A08) of the DFG. The authors are grateful to C. J. Hansen and M. Hanke for the helpful discussions and to the anonymous referee for the constructive suggestions. This work has made use of the SP_Ace spectral analysis tool version 1.2. The data presented herein were obtained at the W.M. Keck Observatory, which is operated as a scientific partnership among the California Institute of Technology, the University of California, and the National Aeronautics and Space Administration. The Observatory was made possible by the generous financial support of the W.M. Keck Foundation. The authors wish to recognize and acknowledge the very significant cultural role and reverence that the summit of Mauna Kea has always had within the indigenous Hawaiian community. We are most fortunate to have the opportunity to conduct observations from this mountain.

\section{References}

Andrievsky, S. M., Spite, M., Korotin, S. A., et al. 2009, A\&A, 494, 1083 Asplund, M., Grevesse, N., Sauval, A. J., \& Scott, P. 2009, ARA\&A, 47, 481 Bastian, N., \& Lardo, C. 2018, ARA\&A, 56, 83

Battistini, C., \& Bensby, T. 2015, A\&A, 577, A9

Battistini, C., \& Bensby, T. 2016, A\&A, 586, A49

Bensby, T., Feltzing, S., \& Oey, M. S. 2014, A\&A, 562, A71

Bergemann, M., \& Cescutti, G. 2010, A\&A, 522, A9

Bergemann, M., Pickering, J. C., \& Gehren, T. 2010, MNRAS, 401, 1334

Boeche, C., \& Grebel, E. K. 2016, A\&A, 587, A2

Bragaglia, A., Carretta, E., D’Orazi, V., et al. 2017, A\&A, 607, A44

Brown, J. A., Wallerstein, G., \& Zucker, D. 1997, AJ, 114, 180

Çalışkan, Ş., Christlieb, N., \& Grebel, E. K. 2012, A\&A, 537, A83

Carollo, D., Beers, T. C., Lee, Y. S., et al. 2007, Nature, 450, 1020

Carretta, E., Bragaglia, A., Gratton, R., \& Lucatello, S. 2009, A\&A, 505, 139

Carretta, E., Bragaglia, A., Lucatello, S., et al. 2017, A\&A, 600, A118

Carretta, E., Bragaglia, A., Lucatello, S., et al. 2018, A\&A, 615, A17

Castelli, F., \& Kurucz, R. L. 2003, IAU Symp., 2010, A20

Catelan, M., Ferraro, F. R., \& Rood, R. T. 2001, ApJ, 560, 970

Cohen, J. G. 2004, AJ, 127, 1545

Cohen, J. G., \& Kirby, E. N. 2012, ApJ, 760, 86

Cohen, J. G., \& Melendez, J. 2005, AJ, 129, 1607

Cooper, A. P., D'Souza, R., Kauffmann, G., et al. 2013, MNRAS, 434, 3348

Cordero, M. J., Hansen, C. J., Johnson, C. I., \& Pilachowski, C. A. 2015, ApJ, 808, L10

Correnti, M., Bellazzini, M., Dalessandro, E., et al. 2011, MNRAS, 417, 2411

Cutri, R. M., Skrutskie, M. F., van Dyk, S., et al. 2003, 2MASS All Sky Catalog of Point Sources

Da Costa, G. S., \& Armandroff, T. E. 1995, AJ, 109, 2533

Dotter, A., Chaboyer, B., Jevremović, D., et al. 2008, ApJS, 178, 89

Dotter, A., Sarajedini, A., \& Anderson, J. 2011, ApJ, 738, 74

Gaia Collaboration (Brown, A. G. A., et al.) 2018, A\&A, 616, A1

Gerashchenko, A. N., \& Ananjevskaja, Y. K. 2018, Astrophysics, 61, 182

Gratton, R. G., Carretta, E., \& Bragaglia, A. 2012, A\&ARv, 20, 50 
Hanke, M., Koch, A., Hansen, C. J., \& McWilliam, A. 2017, A\&A, 599, A97 Hansen, C. J., Primas, F., Hartman, H., et al. 2012, A\&A, 545, A31

Hansen, C. J., Bergemann, M., Cescutti, G., et al. 2013, A\&A, 551, A57

Hansen, C. J., Rich, R. M., Koch, A., et al. 2016a, A\&A, 590, A39

Hansen, C. J., Nordström, B., Hansen, T. T., et al. 2016b, A\&A, 588, A37

Harbeck, D., Smith, G. H., \& Grebel, E. K. 2003, A\&A, 409, 553

Harris, W. E. 1996, AJ, 112, 1487

Hartwick, F. D. A. 1987, in NATO Advanced Science Institutes (ASI) Series C, eds. G. Gilmore, \& B. Carswell, 207, 281

Hendricks, B., Boeche, C., Johnson, C. I., et al. 2016, A\&A, 585, A86

Hilker, M. 2006, A\&A, 448, 171

Johnson, C. I., Rich, R. M., Pilachowski, C. A., et al. 2015, AJ, 150, 63

Johnson, C. I., Caldwell, N., Rich, R. M., \& Walker, M. G. 2017, AJ, 154, 155

Kacharov, N., Koch, A., \& McWilliam, A. 2013, A\&A, 554, A81

Kayser, A., Hilker, M., Grebel, E. K., \& Willemsen, P. G. 2008, A\&A, 486, 437

Kirby, E. N., Guo, M., Zhang, A. J., et al. 2015, ApJ, 801, 125

Kobayashi, C., Umeda, H., Nomoto, K., Tominaga, N., \& Ohkubo, T. 2006, ApJ, 653,1145

Koch, A. 2009, Astron. Nachr., 330, 675

Koch, A., \& Côté, P. 2010, A\&A, 517, A59

Koch, A., \& Côté, P. 2017, A\&A, 601, A41

Koch, A., \& McWilliam, A. 2011, AJ, 142, 63

Koch, A., Rich, R. M., Reitzel, D. B., et al. 2008, ApJ, 689, 958

Koch, A., Côté, P., \& McWilliam, A. 2009, A\&A, 506, 729

Koch, A., McWilliam, A., Preston, G. W., \& Thompson, I. B. 2016, A\&A, 587, A124

Koch, A., Hansen, C. J., \& Kunder, A. 2017, A\&A, 604, A41

Kuzma, P. B., Da Costa, G. S., \& Mackey, A. D. 2018, MNRAS, 473, 2881

Lai, D. K., Rockosi, C. M., Bolte, M., et al. 2009, ApJ, 697, L63

Law, D. R., \& Majewski, S. R. 2010, ApJ, 718, 1128

Lee, J.-W., López-Morales, M., \& Carney, B. W. 2006, ApJ, 646, L119

Lind, K., Asplund, M., Barklem, P. S., \& Belyaev, A. K. 2011a, A\&A, 528, A103

Lind, K., Charbonnel, C., Decressin, T., et al. 2011b, A\&A, 527, A148

Marín-Franch, A., Aparicio, A., Piotto, G., et al. 2009, ApJ, 694, 1498
Marino, A. F., Milone, A. P., Karakas, A. I., et al. 2015, MNRAS, 450, 815 Matteucci, F., \& Brocato, E. 1990, ApJ, 365, 539

Milone, A. P., Piotto, G., Renzini, A., et al. 2017, MNRAS, 464, 3636

Mottini, M., Wallerstein, G., \& McWilliam, A. 2008, AJ, 136, 614

Mucciarelli, A., Bellazzini, M., Ibata, R., et al. 2012, MNRAS, 426, 2889

Mucciarelli, A., Bellazzini, M., Catelan, M., et al. 2013, MNRAS, 435, 3667

Mucciarelli, A., Lapenna, E., Ferraro, F. R., \& Lanzoni, B. 2018, ApJ, 859, 75

Peterson, R. C., \& Latham, D. W. 1989, ApJ, 336, 178

Piatti, A. E., \& Koch, A. 2018, ApJ, 867, 8

Pillepich, A., Madau, P., \& Mayer, L. 2015, ApJ, 799, 184

Placco, V. M., Frebel, A., Beers, T. C., \& Stancliffe, R. J. 2014, ApJ, 797, 21

Pritzl, B. J., Venn, K. A., \& Irwin, M. 2005, AJ, 130, 2140

Pryor, C., \& Meylan, G. 1993, in Structure and Dynamics of Globular Clusters, eds. S. G. Djorgovski, \& G. Meylan, ASP Conf. Ser., 50, 357

Ramírez, I., \& Meléndez, J. 2005, ApJ, 626, 465

Roederer, I. U., Preston, G. W., Thompson, I. B., et al. 2014, AJ, 147, 136

Roederer, I. U., Mateo, M., Bailey, J. I., et al. 2016, MNRAS, 455, 2417

Sbordone, L., Monaco, L., Moni Bidin, C., et al. 2015, A\&A, 579, A104

Searle, L., \& Zinn, R. 1978, ApJ, 225, 357

Sheinis, A. I., Bolte, M., Epps, H. W., et al. 2002, PASP, 114, 851

Shetrone, M. D., Côté, P., \& Sargent, W. L. W. 2001, ApJ, 548, 592

Sneden, C. A. 1973, PhD Thesis, The University of Texas at Austin

Sneden, C., Cowan, J. J., \& Gallino, R. 2008, ARA\&A, 46, 241

Spite, M., Cayrel, R., Plez, B., et al. 2005, A\&A, 430, 655

Stanford, L. M., Da Costa, G. S., \& Norris, J. E. 2010, ApJ, 714, 1001

Stetson, P. B., Bolte, M., Harris, W. E., et al. 1999, AJ, 117, 247

Suntzeff, N., Olszewski, E., \& Stetson, P. B. 1985, AJ, 90, 1481

Timmes, F. X., Woosley, S. E., \& Weaver, T. A. 1995, ApJS, 98, 617

Tolstoy, E., Hill, V., \& Tosi, M. 2009, ARA\&A, 47, 371

Villanova, S., Geisler, D., Carraro, G., Moni Bidin, C., \& Muñoz, C. 2013, ApJ, 778,186

Woosley, S. E., \& Weaver, T. A. 1995, ApJS, 101, 181

Yuan, Z., Smith, M. C., Xue, X. X., et al. 2019, ApJ, submitted [arXiv: 1902.05248] 